\title{
Silicon timing response to different laser light.
}

\author{
Anatoly Ronzhin \\ Fermilab
}

\section{Abstract}

The purpose of the work was to measure pulse shape and timing response of the silicon under picosecond lasers light illumination. Timing response for different laser wavelengths, $405 \mathrm{~nm}, 635 \mathrm{~nm}$ and $1060 \mathrm{~nm}$ was a point of interest. The performed measurements could be useful for CMS HGCal upgrade [1].

Keywords: silicon detectors, time resolution, electrons and holes.

\section{Introduction}

The paper is organized as follows. Setup of the measurement, details of light penetration into $\mathrm{p}$ and $\mathrm{n}$ types of silicon and the silicon parameters described in section 2. Attention was taken to the main source of the time jitter in silicon. Signal's shapes and time resolution (TR) of the silicon were measured and obtained results presented in section 3. Discussion and conclusion presented in sections 4 and 5.

We studied earlier the influence of signal rise time on time resolution (TR) of detectors. The shorter the signal rise time the better time resolution can be obtained [2]. The fact that the rise time of semiconductor detectors is minimal when the detector is totally depleted is well known [3]. The signal rise time is mostly dependent on silicon capacitance, velocity and type of carriers. 
The photons produce high compact ionization in silicon what could lead to fast timing response. Applied bias voltage in silicon is usually high enough $\left(\mathrm{E}>10^{5} \mathrm{~V} / \mathrm{cm}\right)$ to get saturation velocity for carriers [3]. We consider main sources of signal time jitter in silicon under light illumination. Picosecond lasers (PiLas) with wavelength 405nm, $635 \mathrm{~nm}$ and $1060 \mathrm{~nm}$ were used [4]. The absorption length of photons inside of silicon under normal incidence in dependence of the photons wavelength is shown in Fig. 1.

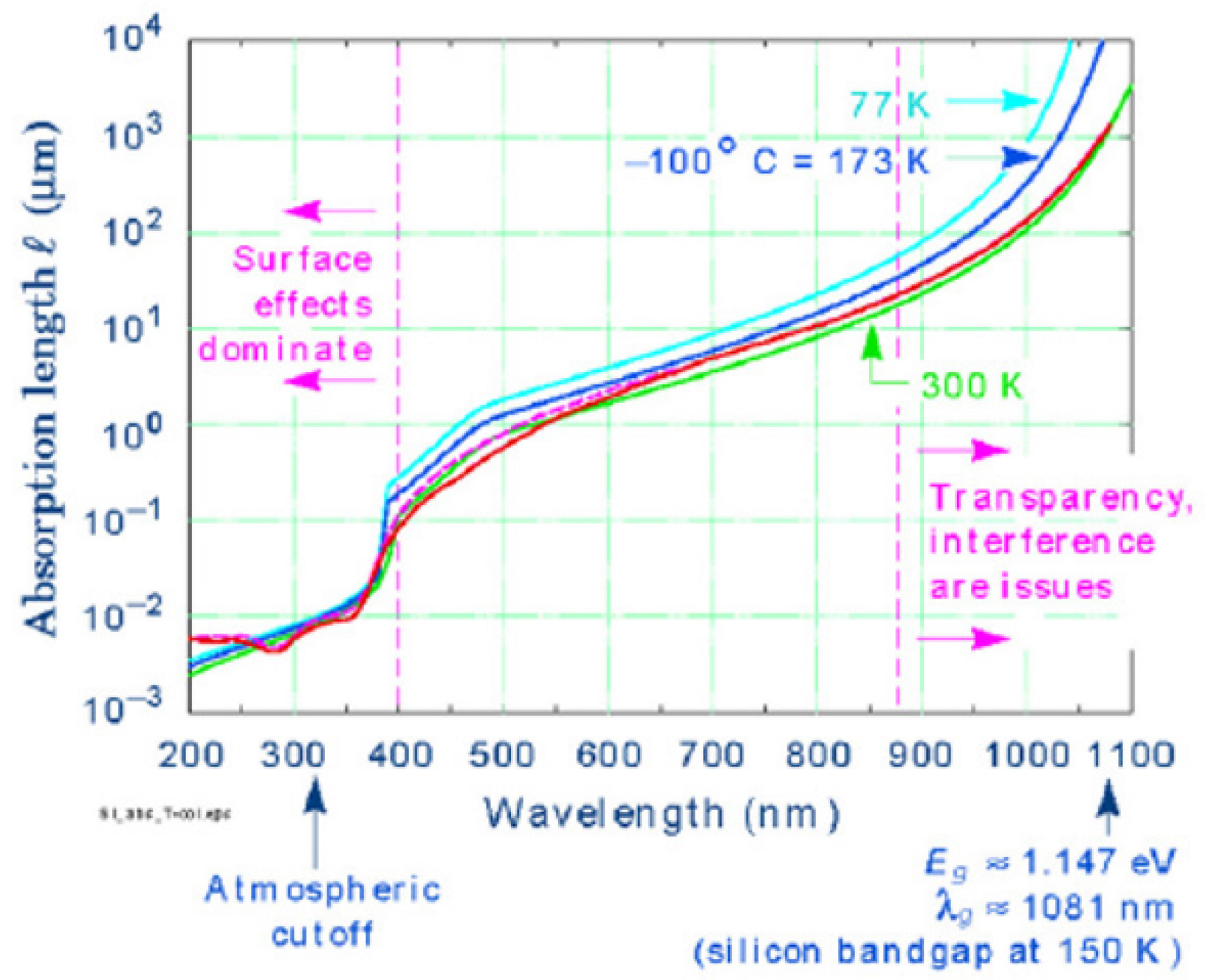

Fig.1. Absorption length of photon in silicon in dependence of its wavelength. 
The aim of this study will be concentrated on details of signals produced by the light with different wavelength, hitting silicon.

The penetration depth of photons inside of silicon is $\sim 100$ $\mathrm{nm}$ for $405 \mathrm{~nm}, 1 \mathrm{um}$ for $635 \mathrm{~nm}$ and $\sim 1,000$ microns for 1060 $\mathrm{nm}$ respectively. One of the goal of the work was to see possible difference in output silicon signal for different laser's wavelengths. Another goal was to study some opportunity to use the lasers for possible detectors calibration [1].

\section{Setup.}

The setup for the measurements is shown in Fig. 2.

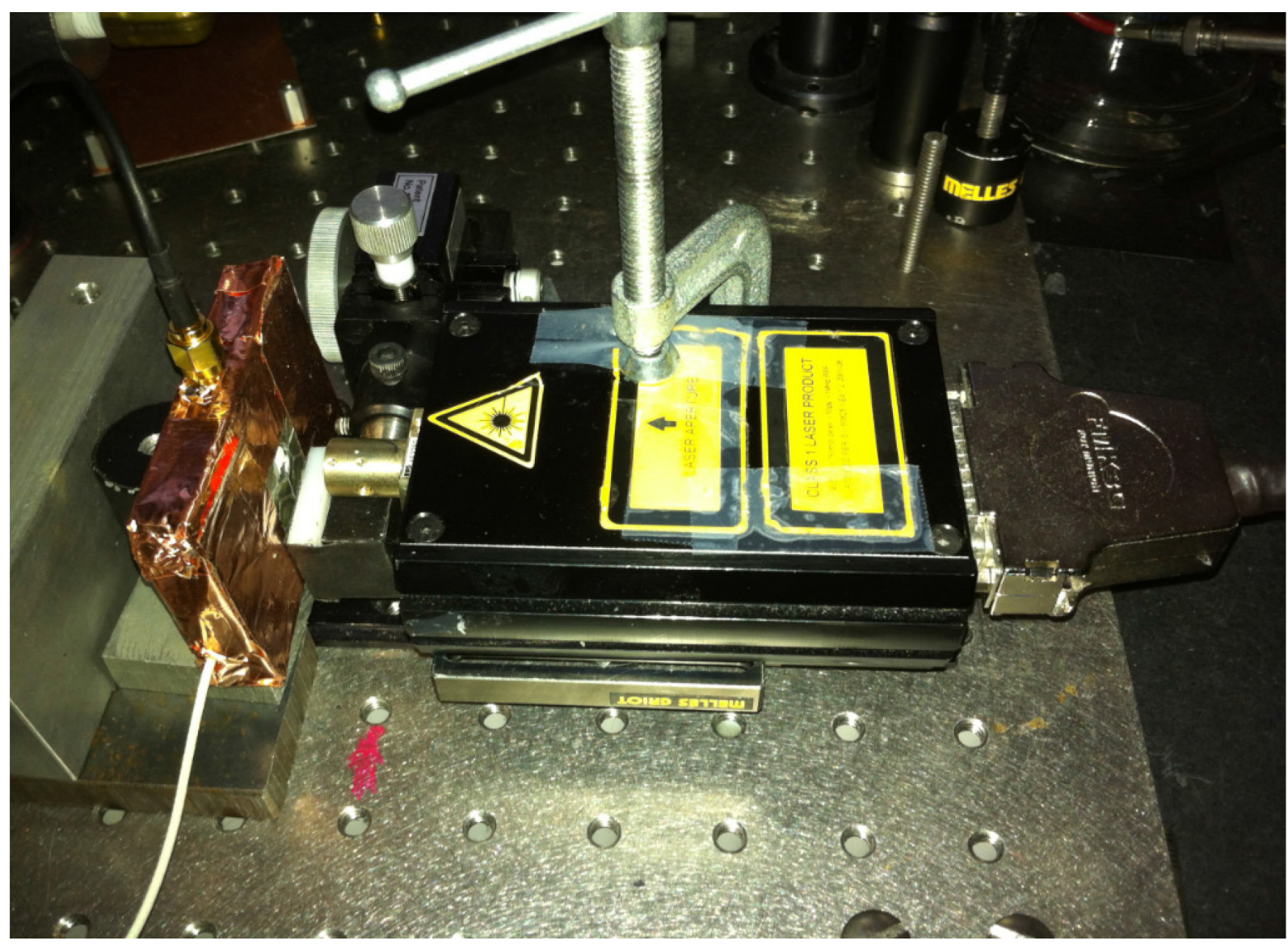

Fig. 2. Laser head and tested silicon inside of copper box 
We used for our test silicon, produced by Hamamatsu [5]. The thickness of the silicon is $\sim 325 \mathrm{um}$. The transverse size of the silicon is $6 \times 6 \mathrm{~mm} 2$. The silicon diode was placed in metal box. The HV was applied to the PC board by the cable terminated with HSV connector. The silicon diode output signal was taken out by using the SMA connector attached to the box. Schematics of the board is shown in Fig. 3. The negative bias voltage was applied to p-side of the silicon. Fig. 4 presents measured capacitance of the diode vs bias voltage.

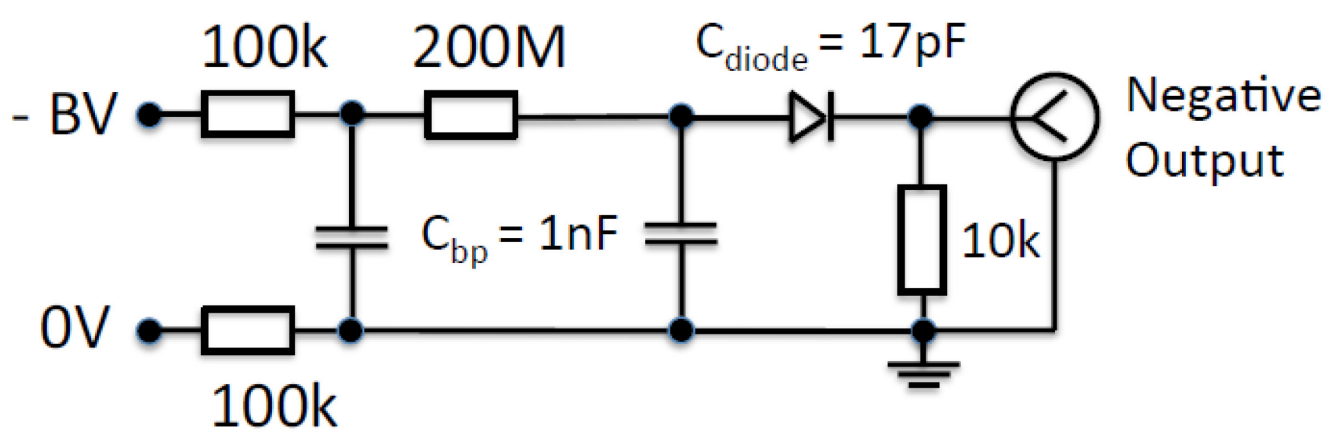

Fig. 3. Electrical schematics of the silicon diode.

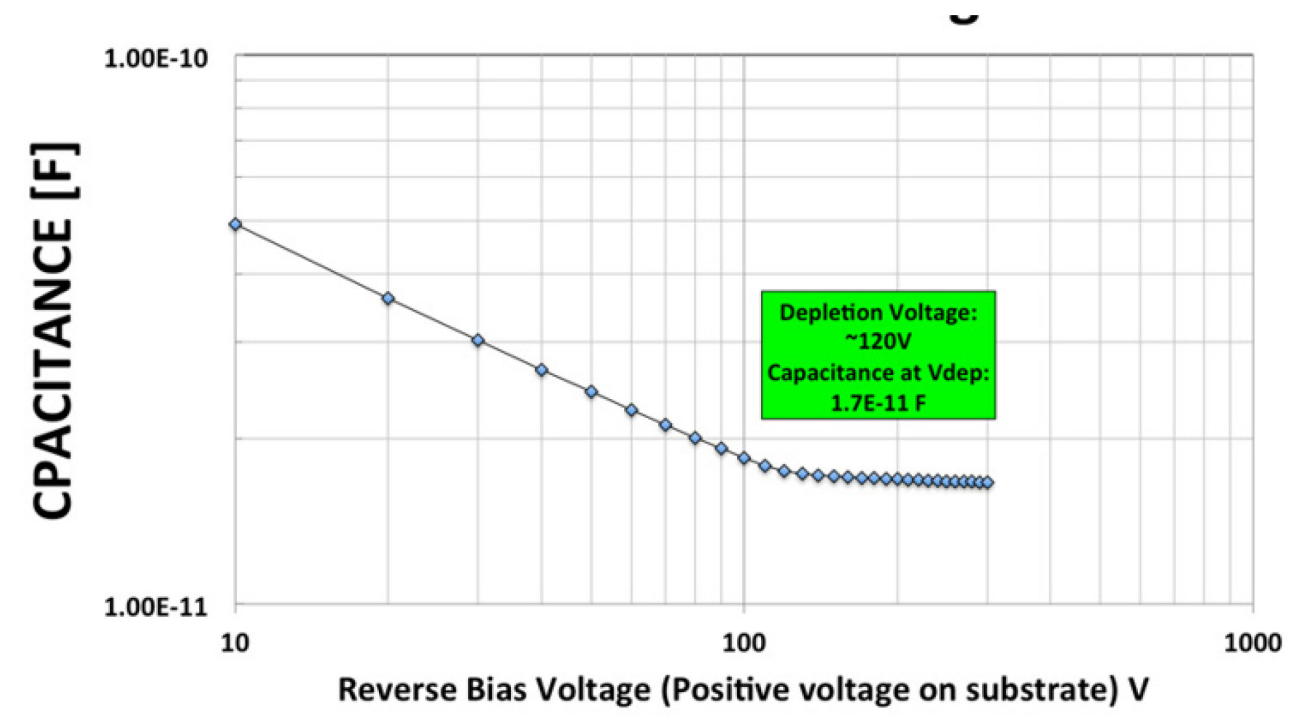

Fig. 4. Diode capacitance vs depletion voltage. 
The junction capacitance depends on the area of p-layer and the thickness of the depletion layer which increased with inverse bias voltage. When photon hits silicon surface it can produce electron hole pairs. The holes collected on the p-side of the silicon and electrons collected on the opposite n-side and forms the output signal. Fig. 5 is shown for illustration.

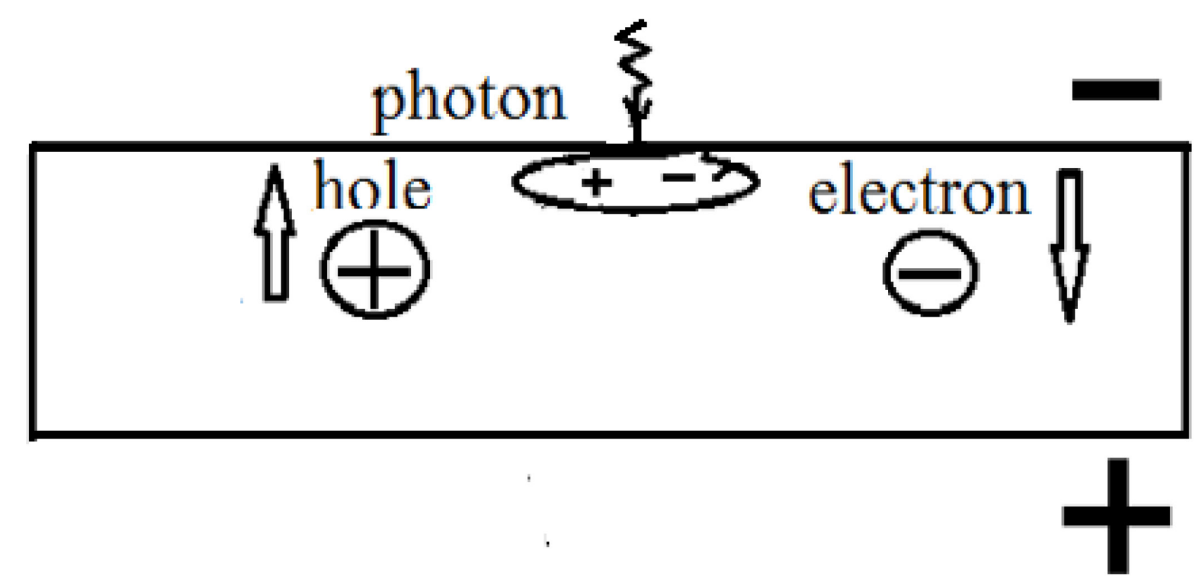

Fig 5, illustration for the carriers collected in silicon.

The density of the produced by light carriers defines the minimum achievable time jitter [2]. The photons produce compact ionization along the direction of the photon incidence. This leads to fast timing response.

\section{Measurements}

DRS4 was used for the readout [6]. The laser light pulse (sigma) was $\sim 15$ ps for all used lasers. The "electrical" time resolution was measured first. The NIM signal was splitted by two halfs and applied to the DRS as "start" and "stop" signals. The obtained time resolution (TR) was $\sim 3.7 \mathrm{ps}$. It is worth to note that the obtained TR allow to measure the difference in the cable length with better of $1 \mathrm{~mm}$ accuracy $(\sim 5 \mathrm{ps} / \mathrm{mm}$ delay in the used cable). Next measurements were performed with lasers 
illuminated the silicon. It was observed some change of the silicon signal shape when bias voltage was changed. Fig. 6 illustrate influence of bias voltage on silicon signal shape (405 $\mathrm{nm}$ light, bias $10 \mathrm{~V}, 300 \mathrm{~V}, 500 \mathrm{~V}$ from left to right). Silicon signal trace is of blue color and trigger signal is rose. The oscillograms were taken by Tektronix TDS 3054B oscilloscop, and the silicon signals were taken without amplifier.
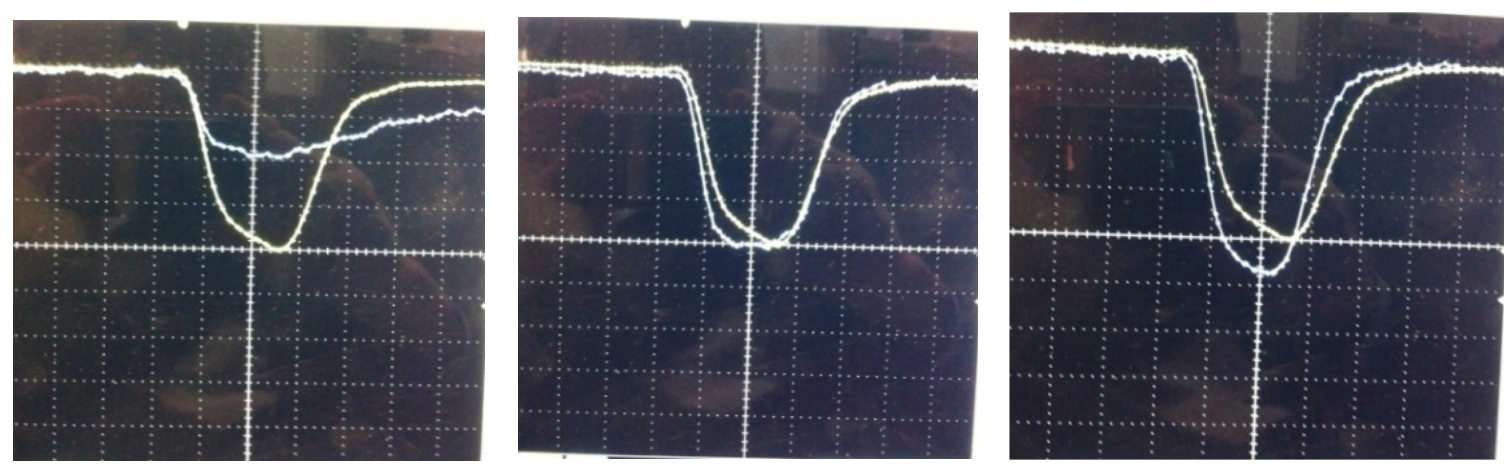

Fig. 6. Traces of triggers and silicon signals. Bias voltage $10 \mathrm{~V}, 300 \mathrm{~V}, 500 \mathrm{~V}$ (from left to right), signals were taken for $405 \mathrm{~nm}$ of the laser light. Almost the same traces obtained for $635 \mathrm{~nm}$ laser light.

The time jitter was measured between the trigger signal and silicon output signal. The silicon signal was amplified by factor $\sim 200$ for the timing measurements. Two Ortec 120 amplifiers in series used in the measurements [7]. The "time stamps" for both trigger and silicon signals was setlled on $\sim 0.5$ of signals amplitude. Dependence of the silicon amplitude and TR on bias voltage was measured for $405 \mathrm{~nm}$ and $1060 \mathrm{~nm}$ of the lasers light, Fig. 7. 


\section{Silicon amplitude, $(\mathrm{mVx} 10)$ and time resolution (TR), ps, vs. Bias, Volts}

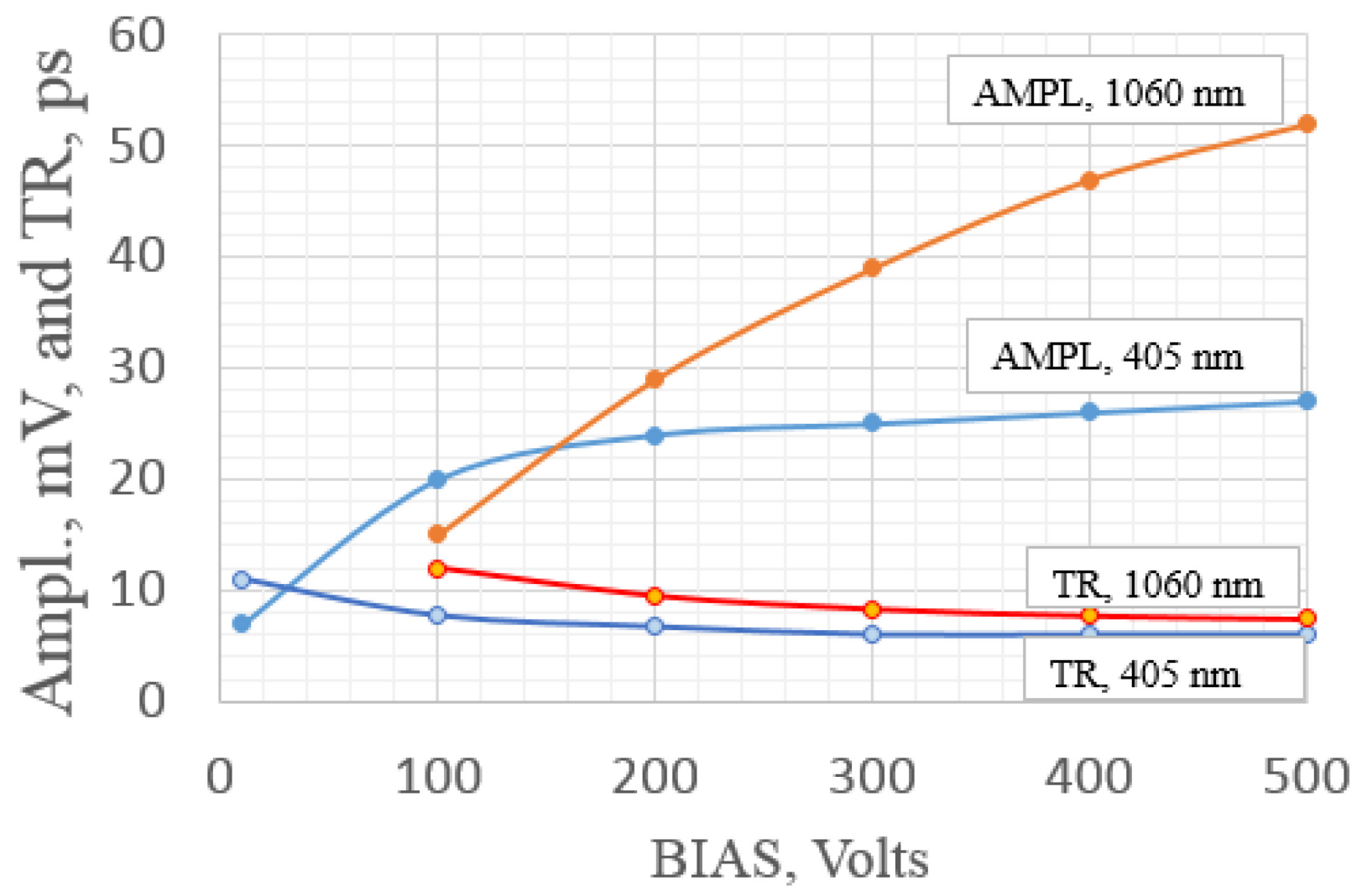

Fig. 7. Time resolution of the silicon in dependence on bias voltage. Wavelength of lasers light was $405 \mathrm{~nm}$ and $1060 \mathrm{~nm}$.

Next series of measurements was performed with $1060 \mathrm{~nm}$ wavelength pilas laser light. The shape of the silicon out put signal was measured in dependence of bias voltage. In Fig. 8 presented traces of signals for bias voltage $10 \mathrm{~V}, 300 \mathrm{~V}$ and 500 $\mathrm{V}$. Time resolution of the silicon in dependence on bias voltage for the laser light $1060 \mathrm{~nm}$ presented in Fig. 7 above. 

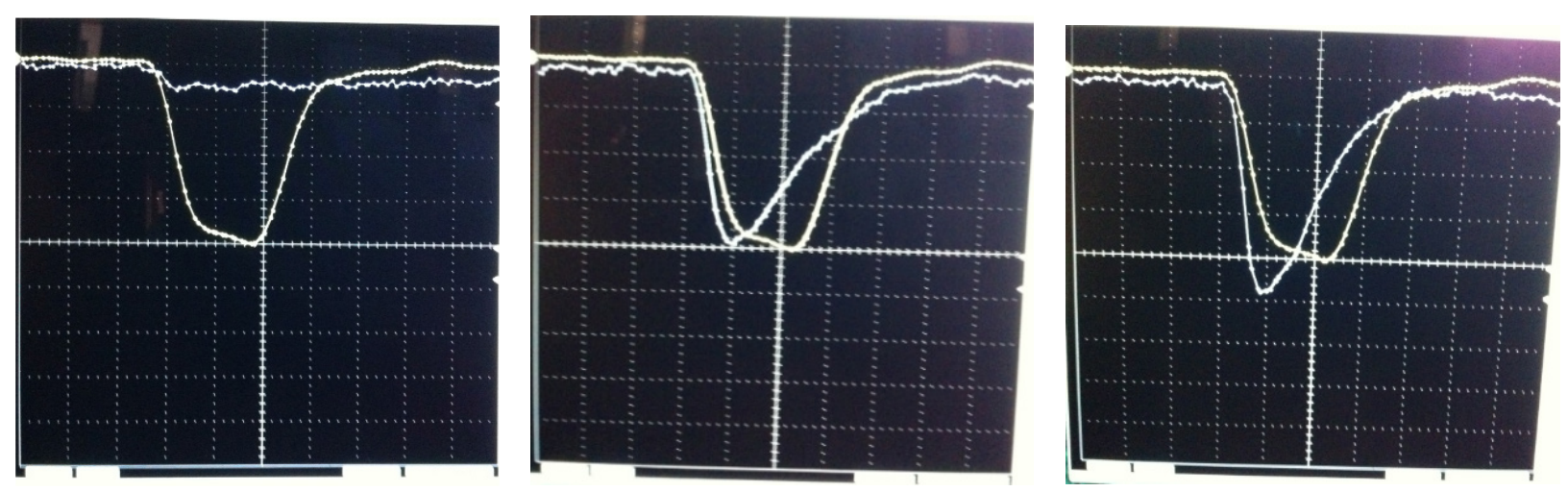

Fig. 8. Traces of triggers ans silicon signals for the 1060 $\mathrm{nm}$ of laser light. Bias voltage $10 \mathrm{~V}, 300 \mathrm{~V}, 500 \mathrm{~V}$ (from left to right).

\section{Discussion}

Fig. 6, 7, 8 show some difference in signal shape and dependence of amplitude on bias voltage when silicon illuminated by $405 \mathrm{~nm}$ and $1060 \mathrm{~nm}$ laser light. The rise time of the signals at full depletion is $\sim 2 \mathrm{~ns}$, but the shape of the signals is a bit different. For $405 \mathrm{~nm}$ the shape is almost symmetrical and for $1060 \mathrm{~nm}$ it is triangle with sharp leading edge and slower decay part. Such a shape could be explaned by different absorption lenths for the $405 \mathrm{~nm}$ and $1060 \mathrm{~nm}$ light. $405 \mathrm{~nm}$ light absorbed in $\sim 100 \mathrm{~nm}$ of the silicon and produces very narrow bucket in direction of incidence. The $1060 \mathrm{~nm}$ light absorbed along all silicon thickness, which is $~ 300$ um, with slower rate than for $405 \mathrm{~nm}$. The different dependences of amplitudes on bias voltage for $405 \mathrm{~nm}$ and $1060 \mathrm{~nm}$ light also can be due to the same reason. 


\section{Conclusion.}

It was not observed big difference in pulse shape of silicon when illuminated by $405 \mathrm{~nm}, 635 \mathrm{~nm}$. and $1060 \mathrm{~nm}$ laser light. The results obtained with PiLas laser illumination of the silicon shows possibility to get few picosecond time resolution (TR). The TR is weakly depends on bias voltage in the range of 50$450 \mathrm{~V}$ of the bias. The best measured TR was $\sim 3.7$ ps with correction on "electronic" time resolution. The result was obtained with maximum Pilas laser light intensity. The TR $=3.7$ ps turned out to be very sensitive to type of cable, the length of the cable, temperature, etc. The TR might be used as a tool to measure such parameters with high accuracy.

The performed measurements show possibility to use the lasers for HGCal calibration [1].

\section{Acknowledgement.}

I would like to express my thanks to S. Los, A. Apresyan, S. Xie and $\mathrm{H}$. Kim for help in some measurements. 


\section{References}

1. J. Butler, D. Contardo, M. Klute, J. Mans, and L. Silvestris, Technical Proposal for the Phase-II Upgrade of the CMS Detector," Tech. Rep. CERN-LHCC-2015-010. LHCC-P-008, CERN, Geneva, Jun 2015.

2. Anatoly Ronzhin. "High time-resolution photodetectors for PET applications". Nuclear Instruments and Methods in Physics Research A809 (2016) 53-57.

3. Helmuth Spieler: Semiconductor Detector Systems, Oxford University Press, 2005.

4. PiLas Laser, Advanced Photonic Systems, A.L.S Gmb. H Schwarzschild str 6, Photonic Center, D-12489 Berlin, Germany.

5. Silicon detectors for high energy particles, chapter 10. https://www.hamamatsu.com/resources/pdf/ssd/e10 handbook_f or high energy.pdf

6. DRS4 Chip Home Page. (http://psi/ch/drs)

7. Ortec VT120C fast preamplifier. www.ortec-online.com 